\title{
A conjecture on the relationship between critical residual entropy and finite temperature pseudo-transitions of one-dimensional models
}

\author{
Onofre Rojas* \\ Departamento de Física, Universidade Federal de Lavras, 37200-900, Lavras - MG, Brazil
}

Abstract

Recently pseudo-critical temperature clues were observed in one-dimensional spin models, such as the Ising-Heisenberg spin models, among others. Here we report a relationship between the zero-temperature phase boundary residual entropy (critical residual entropy) and pseudo-transition. Usually, the residual entropy increases in the phase boundary, which means the system becomes more degenerate at the phase boundary compared to its adjacent states. However, this is not always the case; at zero temperature, there are some phase boundaries where the entropy holds the largest residual entropy of the adjacent states. Therefore, we can propose the following conjecture: If residual entropy at zero-temperature is a continuous function at least from the one-sided limit at a critical point, then pseudo-transition evidence will appear at finite temperature near the critical point. We expect that this argument would apply to study more realistic models. Only by analyzing the residual entropy at zero temperature, one could identify a priori whether the system will exhibit the pseudo-transition at finite temperature. To strengthen our conjecture, we use two examples of Ising-Heisenberg models, which exhibit pseudo-transition behavior: one frustrated coupled tetrahedral chain and another unfrustrated diamond chain.

Keywords: Residual entropy; Quasi-phases; Pseudo-transitions; Ising-Heisenberg

\section{INTRODUCTION}

In 1950, van Hove 1 proposed a theorem to rigorously verify the absence of phase transition with a short-range interaction for a uniform one-dimensional system. This theorem is valid under the following conditions: (i) Homogeneity, excluding automatically inhomogeneous system, i.e., disordered or periodic. (ii) The Hamiltonian does not include particles position terms, e.g., external fields. (iii) Hard-core particles, this means the theorem cannot be applied to point-like or soft particles. The theorem has been proved writing the partition function, by using the transfer-matrix technique and reducing the problem to find the largest eigenvalue, which implies that the free energy is an analytic function. In fact, the theorem proves that the one-dimensional models with short-range coupling do not exhibit any phase transitions. Mermin and Wagner 3] also rigorously proved the absence of ferromagnetism or antiferromagnetism in one and two dimensional isotropic Heisenberg model. Recently, Cuesta and Sanchez[2] proposed a more general non-existence theorem for phase transition at finite temperature. Mainly they included an external field and considered point-like particles, which broadens the nonexistence theorem. But it is not yet a fully general theorem, e.g., no mixed particle chains and more general external fields were included.

Despite of that, there are some one-dimensional models with a short-range coupling that exhibit a first-order phase transition at finite temperature. The Kittel model (also known as the zipper model) [4, is a typical simple model with a finite size transfer-matrix. Note that the constraint on zipper corresponds to an infinite potential, and this condition leads to a non-analytic free

*Electronic address: ors@ufla.br energy. Consequently, the system exhibits a first-order phase transition. Other model is that considered by Chui-Weeks model [5], with a typical set of models called solid-on-solid for surface growth. While in this case the transfer-matrix dimension is infinite, but it can still be solved exactly. Furthermore, imposing the impenetrable condition to subtract, the model shows the existence of phase-transition. Dauxois-Peyrard model [6], is another model with infinite transfer-matrix dimension, which can be solved numerically. More recently Sarkanych et al.7] also proposed a one-dimensional Potts model with invisible states and short-range coupling. The term invisible essentially refers to an additional energy degeneracy, which contributes to the entropy, but not the interaction energy. So, these invisible states are responsible for generating the first-order phase transition. In a nutshell, all these models break the Perron-Frobenius theorem [8], because the free energy becomes non-analytical at the phase transition temperature, or equivalently some elements of transfer-matrix become null (which corresponds to an infinite energy).

On the other hand, the term "pseudo-transition" was introduced by Timonin 9 in 2011 while studying the spin ice in a field, and refers to a sudden change in the first derivative of free energy, whereas a strong vigorous peak appears in the second derivative of free energy, although there are no discontinuity or divergence, respectively. Later this definition was adopted for our group [10 12 because we found the same kind of property. The pseudo-transition does not violate the PerronFrobenius theorem [8], since the free energy is always analytic. The anomalous behavior occurs only because off diagonal elements of the transfer-matrix become a tiny amount compared to other elements.

Recent investigations revealed a number of decorated one-dimensional models, particularly the Ising and Heisenberg models with a variety of structures. Such as the Ising-Heisenberg diamond chain[13, 14] 
and even Ising diamond chain 15. One-dimensional double-tetrahedral model, where the nodal site is assembled by localized Ising spin, and alternating with a pair of delocalized mobile electrons within a triangular plaquette 16. Ladder model with alternating Ising-Heisenberg coupling 17]. As well as the triangular tube model with Ising-Heisenberg coupling [18]. In all aforementioned models, pseudo-transition clues were observed. The first derivative of free energy, such as entropy, internal energy, and magnetization, shows a steep but still continuous change as the temperature varies, which is quite similar to the first-order phase transition behavior. While the second-order derivative of free energy, like the specific heat and magnetic susceptibility, resembles a typical second-order phase transition behavior at finite temperature. Therefore, this peculiar behavior drew attention to a more careful study, as considered in reference [12]. Lately, in reference[10] has been made an additional discussion on this property and detailed study of the correlation function for arbitrarily distant spins around the pseudo-transition.

The article is organized as follows: In sec. 2 we present the free energy for spin-1/2 like one-dimensional model and we analyze the low temperatures behavior. In Sec.3 we present the residual entropy at a critical point, and its connection with the pseudo-transition at finite temperature. In Sec. 4 we apply it to a frustrated Ising-Heisenberg coupled tetrahedral chain. Analogously, in Sec. 5, we also apply it to an unfrustrated Ising-Heisenberg diamond chain. Finally, Sec.6 provides our conclusions and perspectives.

\section{FREE ENERGY}

The models commented above can be seen as decorated models 13, 14, 16, 18, which can be mapped to a simple spin-1/2 Ising-like model 19. Like models considered here [see for instance in eqs. 20 and (37)], can be mapped[19] into an effective Hamiltonian like

$$
H_{\mathrm{eff}}=-\sum_{i=1}^{N}\left[K_{0}+K s_{i} s_{i+1}+\frac{1}{2} B\left(s_{i}+s_{i+1}\right)\right],
$$

where $K_{0}, K$ and $B$ are effective parameters, which may depend on the temperature and original Hamiltonian parameters (for details see for instance ref. [13, 14, 16[18]), assuming the chain contains $N$ unit cells. Therefore, the Hamiltonian (1) transfer-matrix, can be expressed as $\mathbf{V}=\left[\begin{array}{cc}w_{1} & w_{0} \\ w_{0} & w_{-1}\end{array}\right]$, like discussed in reference [12]. The transfer-matrix entries $w_{n}$ (Boltzmann factor) become

$$
w_{n}=\sum_{k=0} g_{n, k} \mathrm{e}^{-\beta \varepsilon_{n, k}}
$$

with $n=\{-1,0,1\}$ (denoted by sectors $-1,0,1$ ). Here $\varepsilon_{n, k}$ represent the energy spectra $k=\{0,1, \ldots\}$ for each sector defined above (not for whole system), and $g_{n, k}$ denotes the degeneracy for each energy level, where we assume $g_{n, k}=\{1,2,3, \ldots\}$. Whereas $\beta=1 / k_{B} T$, with $k_{B}$ being the Boltzmann constant and $T$ is the absolute temperature.

Then the transfer-matrix eigenvalues are provided by

$$
\lambda_{ \pm}=\frac{1}{2}\left(w_{1}+w_{-1} \pm \sqrt{\left(w_{1}-w_{-1}\right)^{2}+4 w_{0}^{2}}\right) .
$$

Assuming the chain has a periodic boundary condition, so the partition function becomes $\mathcal{Z}_{N}=\lambda_{+}^{N}+\lambda_{-}^{N}$. Consequently, the free energy in the thermodynamic limit $(N \rightarrow \infty)$ results in

$$
f=-\frac{1}{\beta} \ln \left[\frac{1}{2}\left(w_{1}+w_{-1}+\sqrt{\left(w_{1}-w_{-1}\right)^{2}+4 w_{0}^{2}}\right)\right] .
$$

\section{A. Low temperature free energy when $w_{0} \rightarrow 0$}

As earlier discussed in the literature[12, if we consider $w_{0}=0$, free energy 4 reduces to

$$
f=-\frac{1}{\beta} \ln \left[\max \left(w_{1}, w_{-1}\right)\right] .
$$

This result could mean the presence of a genuine phase transition at finite temperature, because (5) becomes a non-analytic function when $w_{1}=w_{-1}$. Obviously, this cannot happen in this limit, because $w_{0}$ is small enough but not null.

Now, in general, the energies $\varepsilon_{n, k}$, as defined above, should depend on some parameters, here we simply denote by $x$, e.g., magnetic field or some other parameters. Therefore, let us conveniently define the following quantities: $\bar{\varepsilon}(x)=\frac{\varepsilon_{1,0}(x)+\varepsilon_{-1,0}(x)}{2}$ being the average between lowest energies of both sectors; another quantity we define, is the difference between lowest energies in different sectors $\epsilon(x)=\varepsilon_{1,0}(x)-\varepsilon_{-1,0}(x)$; while $\delta_{1}(x)=\varepsilon_{1,1}(x)-\varepsilon_{1,0}(x)$ and $\delta_{-1}(x)=\varepsilon_{-1,1}(x)-\varepsilon_{-1,0}(x)$ are energy differences within same sector.

Next, let us consider the Boltzmann factor with good accuracy in the low temperatures region, including only the ground state energy and the lowest excited state energy for sectors -1 and 1 . Thus we have

$$
\begin{aligned}
w_{1}(x, T) & =g_{1,0} \mathrm{e}^{-\beta \varepsilon_{1,0}(x)}+g_{1,1} \mathrm{e}^{-\beta \varepsilon_{1,1}(x)} \\
& =\mathrm{e}^{-\beta \bar{\varepsilon}(x)} \eta_{1}(x, T), \\
w_{-1}(x, T) & =g_{-1,0} \mathrm{e}^{-\beta \varepsilon_{-1,0}(x)}+g_{-1,1} \mathrm{e}^{-\beta \varepsilon_{-1,1}(x)} \\
& =\mathrm{e}^{-\beta \bar{\varepsilon}(x)} \eta_{-1}(x, T),
\end{aligned}
$$

where we define

$$
\eta_{\nu}(x, T)=g_{\nu, 0} \mathrm{e}^{-\nu \frac{\epsilon(x)}{2 T}}\left[1+\frac{g_{\nu, 1}}{g_{\nu, 0}} \mathrm{e}^{-\frac{\delta_{\nu}(x)}{T}}\right]
$$

with $\nu= \pm 1$. 
The free energy (5) can be rewritten in the neighboring of quantum phase transition as a function of temperature $T$ and parameter $x$,

$$
f(x, T)=\bar{\varepsilon}(x)-T \ln \left\{\max \left[\eta_{1}(x, T), \eta_{-1}(x, T)\right]\right\} .
$$

From now on, we will focus on the thermodynamic properties when $w_{1}$ and $w_{-1}$ are competing terms and under the condition $w_{0} \rightarrow 0$.

\section{RESIDUAL ENTROPY AND PSEUDO- TRANSITION}

In this section, we will discuss residual entropy at zero temperature, and particularly we stress residual entropy around the ground state phase transition. This phase transition will occur by varying some control parameter like $x$, at a critical point $x_{c}$. The entropy, at this point, will simply be called critical residual entropy (CRE). At lats, we observe that CRE has a relationship with the finite temperature pseudo-transition [20].

\section{A. On the continuity of residual entropy}

At zero temperature, the critical residual entropy (CRE) occurs at the phase boundary of two ground states. For this purpose, we can use the free energy given in (4) when $T \rightarrow 0$. Hence, varying a given parameter $x$, we can get the residual entropy at zero temperature. Considering that the zero temperature phase transition arises at $x_{c}$, then adjacent states coexist, where we denote the critical energy $\varepsilon_{c}$ and corresponding critical degeneracy defined by $G_{c}$.

Now let us continue assuming the condition of the previous section, that is $w_{0} \rightarrow 0$, while $w_{1}$ and $w_{-1}$ are competing terms. Thus, the lowest energies for each sectors are $\varepsilon_{1,0}(x)$ and $\varepsilon_{-1,0}(x)$, in the interface we have $\varepsilon_{1,0}\left(x_{c}\right)=\varepsilon_{-1,0}\left(x_{c}\right)=\varepsilon_{c}$. For a particular case when $x \rightarrow x_{c}$, we have the following limits in the expression (8): $\lim _{x \rightarrow x_{c}} \frac{\delta_{ \pm 1}(x)}{T} \rightarrow+\infty, \lim _{x \rightarrow x_{c}} \epsilon(x)=0$ and $\lim _{x \rightarrow x_{c}} \varepsilon(x)=\varepsilon_{c}$. So the free energy (9) reduces to

$$
f\left(x_{c}, T\right)=\varepsilon_{c}-T \ln \left[\max \left(g_{1,0}, g_{-1,0}\right)\right] .
$$

Afterward, we can obtain the corresponding CRE at zero temperature

$$
\mathcal{S}_{c}=\ln \left[\max \left(g_{1,0}, g_{-1,0}\right)\right] .
$$

Through this article we will consider the entropy in units of $k_{B}$. Moreover, the critical degeneracy per unit cell results in $G_{c}=\max \left(g_{1,0}, g_{-1,0}\right)$.

It is worth mentioning, according to the third law of thermodynamics or often referred to as the Nernst's postulate. At zero temperature, entropy leads to a constant and must be independent of any parameter ( $\operatorname{such}$ as $x$ ),
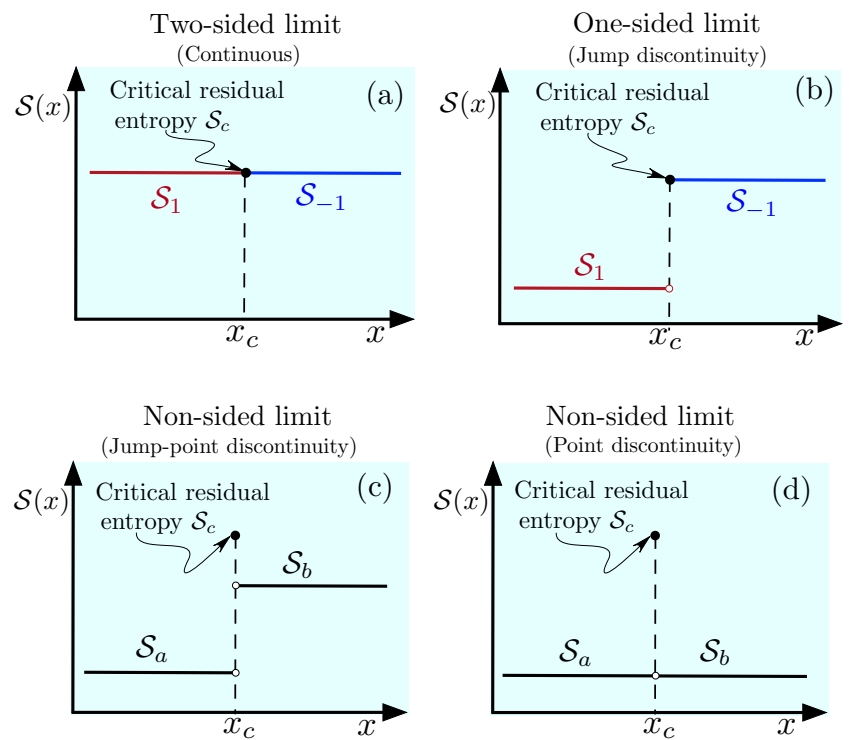

Figure 1: (a) Typical continuous residual entropy at zero temperature as a function of parameter $x$, assuming the system has two phases; (b) Continuous residual entropy at zero temperature from the one-sided limit at $x_{c} ;(\mathrm{c}-\mathrm{d})$ Typical discontinuous residual entropy from the non-sided limit at $x_{c}$, the $\mathrm{CRE}$ is strictly larger than neighboring residual entropy.

so residual entropy is determined only by its ground state energy degeneracy.

Below we present an accurate mathematical formulation[21] of the residual entropy around the critical point found in 11 .

When $g_{1,0}=g_{-1,0}$, residual entropy is illustrated schematically in fig 1 a. Therefore, entropy as a function of $x$ at zero temperature, has left and right limits,

$$
\lim _{x \rightarrow x_{c}^{-}} \mathcal{S}(x)=\lim _{x \rightarrow x_{c}^{+}} \mathcal{S}(x)=\mathcal{S}\left(x_{c}\right)=\mathcal{S}_{c},
$$

and both limits are identical, then we say the residual entropy is continuous at $x_{c}$. However, it is worth mentioning that we are considering two different adjacent phases, which physically correspond to two different states, with identical residual entropies (12). We will see this case later when we apply it to a peculiar unfrustrated model.

When $g_{1,0} \neq g_{-1,0}$, residual entropy is illustrated schematically in fig $1 \mathrm{~b}$. Assuming that the degeneracies of the adjacent phases satisfy $g_{-1,0}<g_{1,0}$, then the left and right limit of the residual entropy at $x_{c}$ becomes,

$$
\lim _{x \rightarrow x_{c}^{-}} \mathcal{S}(x)<\lim _{x \rightarrow x_{c}^{+}} \mathcal{S}(x)=\mathcal{S}\left(x_{c}\right)=\mathcal{S}_{c} .
$$

In this case, the residual entropy is continuous from the right-sided limit, but discontinuous from the left-sided limit 21]. Consequently, we can say that the residual entropy is continuous from the one-sided limit at $x_{c}$. Opposite condition $g_{-1,0}>g_{1,0}$, can be obtained in a similar way.

On the other hand, in Appendix A, we present some detailed results for discontinuous residual entropy, 
where we considered a number of combinations among other sectors. In all those cases, critical degeneracy is strictly larger than the surrounding degeneracy $G_{c}>$ $\max \left(g_{1,0}, g_{-1,0}\right)$.

Therefore, residual entropy must satisfy the following relation

$$
\lim _{x \rightarrow x_{c}^{ \pm}} \mathcal{S}(x)<\mathcal{S}\left(x_{c}\right)=\mathcal{S}_{c}
$$

which is discontinuous at $x_{c}$. Because there is no left or right sided limit 21], so it has a jump-point (fig,1 1 ) and point (fig $1 \mathrm{~d}$ ) discontinuity at $x_{c}$. The fig, $1 \mathrm{~d}$ cannot be confused with a "removable" point discontinuity, because the CRE is strictly larger than the neighboring residual entropy. Note that $\mathcal{S}_{a}$ and $\mathcal{S}_{b}$ in fig,1 -d can represent either $\mathcal{S}_{1}$ or $\mathcal{S}_{-1}$.

\section{B. Pseudo-critical temperature}

As discussed previously in ref. [10, 12, the pseudocritical temperature can be found using the following relation

$$
w_{1}\left(x_{p}, T_{p}\right)=w_{-1}\left(x_{p}, T_{p}\right)
$$

where $x_{p}$ and $T_{p}$ correspond to the Hamiltonian parameters at the pseudo-transition point.

As a first approximation, we can consider only the lowest energy for each sector $n=1$ and -1 . Thereby, the Boltzmann factors become $w_{1}=g_{1,0} \mathrm{e}^{-\beta \varepsilon_{1,0}}$ and $w_{-1}=g_{-1,0} \mathrm{e}^{-\beta \varepsilon_{-1,0}}$.

Besides assuming $\epsilon_{p}=\epsilon\left(x_{p}\right)$ in 15 , we obtain the following relation,

$$
\mathrm{e}^{-\frac{\epsilon_{p}}{T_{p}}}=\frac{g_{-1,0}}{g_{1,0}}
$$

Then we get a simple expression for the pseudo-critical temperature

$$
T_{p}=\frac{\epsilon_{p}}{\ln \left(\frac{g_{1,0}}{g_{-1,0}}\right)}=\frac{\varepsilon_{1,0}\left(x_{p}\right)-\varepsilon_{-1,0}\left(x_{p}\right)}{\ln \left(\frac{g_{1,0}}{g_{-1,0}}\right)} .
$$

Note that result (17) has already been discussed in reference 16-18. It is also worth mentioning that the critical temperature for the Kittel model[4] has a quite similar expression.

However, eq. (17) fails in the case of $g_{1,0}=g_{-1,0}$ because $T_{p}$ turns undefined. To improve 117, we need to include the lowest excited energy in at least one sector. Hence, using eq. (8) in eq. 15), we obtain

$$
\eta_{1}\left(x_{p}, T_{p}\right)=\eta_{-1}\left(x_{p}, T_{p}\right)
$$

Even more explicitly, we can write in terms of a transcendental equation as follows

$$
\begin{aligned}
\mathrm{e}^{-\frac{\epsilon_{p}}{T_{p}}} & =\frac{g_{-1,0}+g_{-1,1} \mathrm{e}^{-\frac{\delta_{-1, p}}{T_{p}}}}{g_{1,0}+g_{1,1} \mathrm{e}^{-\frac{\delta_{1, p}}{T_{p}}}}, \\
& =\left(\frac{g_{-1,0}}{g_{1,0}}\right) \frac{1+\frac{g_{-1,1}}{g_{-1,0}} \mathrm{e}^{-\frac{\delta_{-1, p}}{T_{p}}}}{1+\frac{g_{1,1}}{g_{1,0}} \mathrm{e}^{-\frac{\delta_{1, p}}{T_{p}}}},
\end{aligned}
$$

where $\epsilon_{p}=\epsilon\left(x_{p}\right), \delta_{1, p}=\delta_{1}\left(x_{p}\right)$ and $\delta_{-1, p}=\delta_{-1}\left(x_{p}\right)$ are the aforementioned energy differences.

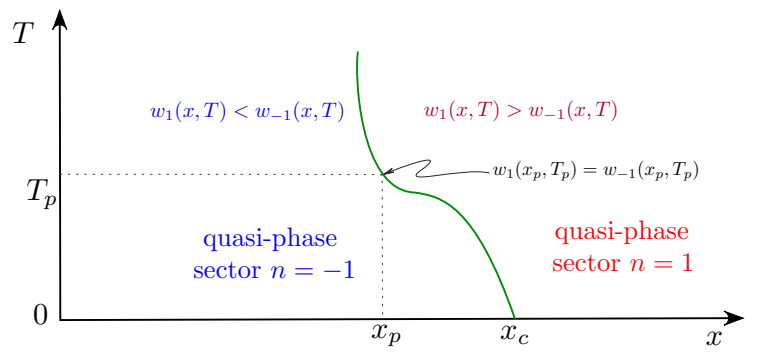

Figure 2: Typical quasi-phase 9 diagram $x$ against $T$. Real phase transition occurs only at zero temperature $\left(x_{c}, 0\right)$. For finite temperature $(T>0)$ arises a pseudo-transition at $\left(x_{p}, T_{p}\right)$.

Usually, when the degeneracies in the ground states are unequal, it is enough to use (17). Nevertheless, when the ground state degeneracies are identical, we need to use eq. 19. Surely, we can also use the full expression given by (15), which we can readily solve by numerical computation.

In fig, 2 we schematically illustrate a typical pseudotransition curve given by eq. 15. Here, we remark that a true phase transition occurs only at zero temperature and for a given critical point $x_{c}$.

In ref. 22, a similar approach was considered when analyzing the maximum peak for the specific heat, and the peak height is related to the degeneracy of the ground state energy.

In summary, the most interesting result from the above study leads to the following conjecture: If zerotemperature residual entropy is continuous at a critical point at least from the one-sided limit, then we can observe vestiges of finite temperature pseudo-transition near the critical point.

Residual entropy, as illustrated in fig 1 a is a differentiable two-sided function[21] at a critical point (12). Due to the differentiability of residual entropy, as soon as temperature increases, entropy should increase smoothly around $x_{c}$, bringing relevant information from adjacent ground state phases without significant disturbances.

Similarly, residual entropy described by fig $1 \mathrm{~b}$ is a one-sided differentiable function at a critical point 13 . Therefore, as in the previous case, the one-sided differentiability takes into account relevant information about 
the adjacent ground states, which should persist without significant disturbance as temperature increases.

In contrast, the discontinuous entropy given by eq. (14) is a non-differentiable function at a critical point. Therefore, we observe as soon as the temperature increases, the adjacent phases near the critical point stretched around critical residual entropy (single point), destroying any evidence of zero-temperature phase transition as temperature increases. So we do not observe a pseudo-transition at finite temperature.

\section{ISING-HEISENBERG COUPLED TETRAHE- DRAL CHAIN}

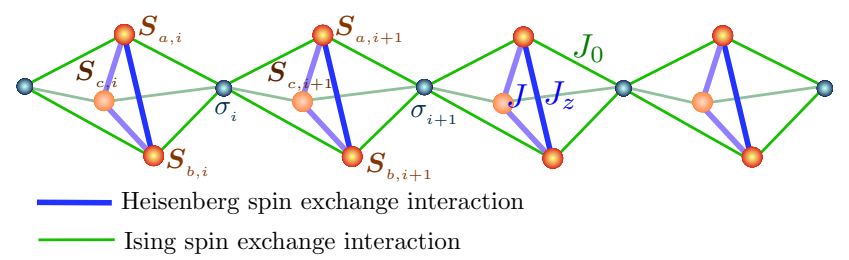

Figure 3: Schematic representation of Ising-Heisenberg coupled tetrahedral chain. Small spheres $\left(\sigma_{i}\right)$ corresponds to Ising spins, and large spheres $\left(\boldsymbol{S}_{a(b), i}\right)$ correspond to Heisenberg spins.

Earlier, in the reference [23, 24], the Heisenberg version of the coupled tetrahedral chain was investigated. While Galisova and Strecka [16, 25], considered delocalized electrons and Ising spin in the tetrahedral chain. Later, in the reference [26, 27] the Ising-Heisenberg version of the model was introduced. Similar model with higher spin was also analyzed more recently in reference [28].

Here we explore a slightly different model (see fig 3), which exhibits a pseudo-transition property that has not yet been discussed. So, we present the Hamiltonian of the model as follows

$$
\begin{aligned}
H= & -\sum_{i=1}^{N}\left\{J\left(\boldsymbol{S}_{a, i}, \boldsymbol{S}_{b, i}\right)_{z}+J\left(\boldsymbol{S}_{b, i}, \boldsymbol{S}_{c, i}\right)_{z}\right. \\
& +J\left(\boldsymbol{S}_{c, i}, \boldsymbol{S}_{a, i}\right)_{z}+\frac{h}{2}\left(\sigma_{i}+\sigma_{i+1}\right) \\
& \left.+\left(S_{a, i}^{z}+S_{b, i}^{z}+S_{c, i}^{z}\right)\left[h_{z}+J_{0}\left(\sigma_{i}+\sigma_{i+1}\right)\right]\right\}
\end{aligned}
$$

where $J\left(\boldsymbol{S}_{a, i}, \boldsymbol{S}_{b, i}\right)_{z}=J S_{a, i}^{x} S_{b, i}^{x}+J S_{a, i}^{y} S_{b, i}^{y}+J_{z} S_{a, i}^{z} S_{b, i}^{z}$, with $S_{a, i}^{\alpha}$ denoting the Heisenberg spin-1/2, and $\alpha=$ $\{x, y, z\}$, while $\sigma_{i}$ denotes the Ising $\operatorname{spin}\left(\sigma_{i}= \pm \frac{1}{2}\right)$. In a similar way we define for sites $b$ and $c$ in 20 . While $J$ describes the exchange interaction of Heisenberg spin in $x y$ anisotropy, similarly $J_{z}$ stands for $z$-anisotropy exchange interaction, and by $J_{0}$ we denote the Ising-Heisenberg exchange interaction. Whereas $h$ and $h_{z}$ correspond to the magnetic field acting on the Ising and Heisenberg spins.

Within the triangle structure, Heisenberg spins must compose the $8 \times 8$ dimension operator. But this operator we can express as block matrices, one quadruplet and two doublet states, which can be readily diagonalized. The quadruplet have two eigenvalues: the first one is

$$
e_{\frac{3}{2}, \frac{3}{2}}=-\frac{3 J_{z}}{4}
$$

with corresponding eigenvectors

$$
\left|\frac{3}{2},+\frac{3}{2}\right\rangle=\left|\begin{array}{l}
+ \\
+ \\
+
\end{array}\right\rangle \text { and }\left|\frac{3}{2},-\frac{3}{2}\right\rangle=\left|\begin{array}{l}
- \\
- \\
-
\end{array}\right\rangle \text {; }
$$

the second eigenvalue is

$$
e_{\frac{3}{2}, \frac{1}{2}}=-J+\frac{J_{z}}{4},
$$

whose eigenvectors are given by

$$
\begin{aligned}
& \left|\frac{3}{2},+\frac{1}{2}\right\rangle=\frac{1}{\sqrt{3}}\left(\left|\begin{array}{l}
+ \\
+ \\
-
\end{array}\right\rangle+\left|\begin{array}{l}
+ \\
\frac{+}{+}
\end{array}\right\rangle+\left|\begin{array}{l}
- \\
+ \\
+
\end{array}\right\rangle\right), \\
& \left|\frac{3}{2},-\frac{1}{2}\right\rangle=\frac{1}{\sqrt{3}}\left(\left|\begin{array}{l}
- \\
- \\
+
\end{array}\right\rangle+\left|\begin{array}{l}
- \\
+ \\
-
\end{array}\right\rangle+\left|\begin{array}{l}
+ \\
- \\
-
\end{array}\right\rangle\right)
\end{aligned}
$$

both energy levels are twofold degenerate.

While the eigenvalue of the doublet pair is

$$
e_{\frac{1}{2}, \frac{1}{2}}=\frac{J}{2}+\frac{J_{z}}{4}
$$

this energy level is fourfold degenerate.

The first doublet eigenstates become

$$
\left\{\begin{array}{l}
\left.\left|\frac{1}{2},+\frac{1}{2}\right\rangle=\frac{1}{\sqrt{6}}\left(\left|\begin{array}{l}
+ \\
+ \\
-
\end{array}\right\rangle-2\left|\begin{array}{l}
+ \\
- \\
+ \\
+
\end{array}\right\rangle-\left|\begin{array}{l}
+ \\
+ \\
+
\end{array}\right\rangle\right),\left|\begin{array}{l}
- \\
+ \\
+
\end{array}\right\rangle\right), \\
\left.\left|\frac{1}{2},+\frac{1}{2}\right\rangle=\frac{1}{\sqrt{2}}\right),
\end{array}\right.
$$

while the second doublet eigenstates are

$$
\left\{\begin{array}{l}
\left.\left|\frac{1}{2},-\frac{1}{2}\right\rangle=\frac{1}{\sqrt{6}}\left(\left|\begin{array}{l}
- \\
- \\
+
\end{array}\right\rangle-2\left|\begin{array}{l}
- \\
- \\
-
\end{array}-\right| \begin{array}{l}
+ \\
-
\end{array}\right\rangle-\left|\begin{array}{l}
+ \\
-
\end{array}\right\rangle\right) \\
\left|\frac{1}{2},-\frac{1}{2}\right\rangle=
\end{array}\right.
$$

\section{A. Zero temperature phase diagram}

Now using the eigenvalues found above, we can express the energy levels and corresponding degeneracies,

$$
\begin{array}{ll}
\varepsilon_{n, 0}=\frac{1}{2}\left(J_{0}-h\right) n-J+\frac{J_{z}}{4}+\frac{h_{z}}{2}, & g_{n, 0}=1, \\
\varepsilon_{n, 1}=\frac{1}{2}\left(3 J_{0}-h\right) n-\frac{3 J_{z}}{4}+\frac{3 h_{z}}{2}, & g_{n, 1}=1, \\
\varepsilon_{n, 2}=\frac{1}{2}\left(J_{0}-h\right) n+\frac{J}{2}+\frac{J_{z}}{4}+\frac{h_{z}}{2}, & g_{n, 2}=2, \\
\varepsilon_{n, 3}=-\frac{1}{2}\left(J_{0}+h\right) n+\frac{J}{2}+\frac{J_{z}}{4}-\frac{h_{z}}{2}, & g_{n, 3}=2, \\
\varepsilon_{n, 4}=-\frac{1}{2}\left(3 J_{0}+h\right) n-\frac{3 J_{z}}{4}-\frac{3 h_{z}}{2}, & g_{n, 4}=1, \\
\varepsilon_{n, 5}=-\frac{1}{2}\left(J_{0}+h\right) n-J+\frac{J_{z}}{4}-\frac{h_{z}}{2}, & g_{n, 5}=1,
\end{array}
$$


(a)
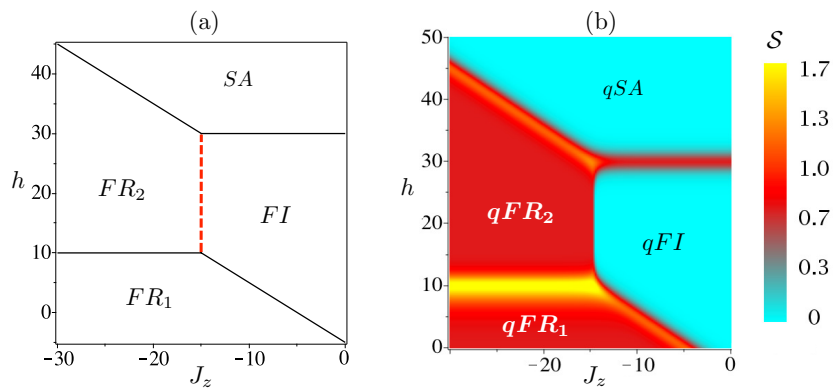

Figure 4: (a) Zero temperature the phase diagram for IsingHeisenberg coupled tetrahedral chain in the $J_{z}-h$ plane, assuming the fixed parameters $J=-10, J_{0}=-10$ and $h_{z}=$ $h$; (b) Entropy density plot for temperature $T=0.6$, assuming the same set of parameters considered in (a).

where $n=\{-1,0,1\}$. To analyze the phase diagram at zero temperature, we explore some relevant states below.

First, we report the ground state energy of the saturated phase $(\mathrm{SA})$, which reads as

$$
E_{S A}=\varepsilon_{1,4}=-\frac{1}{2}\left(3 J_{0}+h\right)-\frac{3 J_{z}}{4}-\frac{3 h_{z}}{2} .
$$

While its ground state becomes

$$
|S A\rangle=\prod_{i=1}^{N}\left|\frac{3}{2},+\frac{3}{2}\right\rangle_{i}|\uparrow\rangle_{i},
$$

whose Ising spin magnetization per unit cell is $m_{I}=\frac{1}{2}$, and Heisenberg spin magnetization per unit cell is $m_{H}=$ $\frac{3}{2}$, with total spin magnetization per unit cell $m_{t}=m_{I}+$ $m_{H}=2$.

Second, the ground state energy for ferrimagnetic (FI) phase can be expressed as

$$
E_{F I}=\varepsilon_{-1,4}=\frac{1}{2}\left(3 J_{0}+h\right)-\frac{3 J_{z}}{4}-\frac{3 h_{z}}{2},
$$

and its ground state and magnetizations turns in,

$$
|F I\rangle=\prod_{i=1}^{N}\left|\frac{3}{2},+\frac{3}{2}\right\rangle_{i}|\downarrow\rangle_{i},
$$

with $m_{I}=-\frac{1}{2}, m_{H}=\frac{3}{2}$ and $m_{t}=1$.

The third phase we consider is a frustrated phase, whose ground state energy is given by

$$
E_{F R_{1}}=\varepsilon_{1,2}=\frac{1}{2}\left(J_{0}-h\right)+\frac{J}{2}+\frac{J_{z}}{4}+\frac{h_{z}}{2},
$$

with corresponding ground state

$$
\left|F R_{1}\right\rangle=\prod_{i=1}^{N}\left|\frac{1}{2},-\frac{1}{2}\right\rangle_{i}|\uparrow\rangle_{i},
$$

whose magnetizations are $m_{I}=\frac{1}{2}, m_{H}=-\frac{1}{2}$ and $m_{t}=$ 0 .
Fourth, one additional frustrated ground state energy is considered

$$
E_{F R_{2}}=\varepsilon_{1,3}=-\frac{1}{2}\left(J_{0}+h\right)+\frac{J}{2}+\frac{J_{z}}{4}-\frac{h_{z}}{2},
$$

and its respective ground state is represented by

$$
\left|F R_{2}\right\rangle=\prod_{i=1}^{N}\left|\frac{1}{2},+\frac{1}{2}\right\rangle_{i}|\uparrow\rangle_{i},
$$

with magnetizations $m_{I}=\frac{1}{2}, m_{H}=\frac{1}{2}$ and $m_{t}=1$.

In fig $4 \mathrm{a}$, the phase diagram is shown at zero temperature, where ground state is described in each region. The phase boundary between $F R_{1}$ and $F R_{2}$ is given by $h=10$, whose phase boundary degeneracy is composed by $g_{1,0}^{c}=2, g_{1,1}^{c}=2, g_{-1,0}^{c}=2$ and $g_{0,0}^{c}=2$, then using the eq. A6 , the CRE becomes $\mathcal{S}_{c}=\ln (3+\sqrt{5})$. The straight line describing the interface between $F R_{1}$ and $F I$ is given by $h=-J_{z}+0.5$. Hence, the CRE can be obtained using eq. A2 , so we have $\mathcal{S}_{c}=\ln (3)$, because $g_{1,0}^{c}=2$ and $g_{-1,1}^{c}=1$. In a similar way, the boundary between $F I$ and $S A$ is given by $h=30$. Thus we can obtain residual entropy using eq. A6 , which becomes $\mathcal{S}_{c}=\ln (2)$, since $g_{1,0}^{c}=1$ and $g_{-1,0}^{c}=1$. Another case, is the boundary between $S A$ and $F R_{2}$ given by $h=-J_{z}-1$. The CRE at zero temperature can be obtained using the eq. A4, where residual entropy becomes $\mathcal{S}_{c}=\ln (3)$, because $g_{1,0}^{c}=2$ and $g_{1,1}^{c}=1$. All the above phase boundaries are clearly discontinuous at phase boundary (14), indicating the absence of the pseudo-transition (see fig 1 p). In contrast, the phase boundary between $F I$ and $F R_{2}$ is described by $J_{z}=-15$ (red dashed line). The CRE satisfying the relation (11) becomes $\mathcal{S}_{c}=\ln (2)$, since the adjacent phases degeneracies are given by $g_{1.0}^{c}=2$ and $g_{-1,0}^{c}=1$, which is in accordance with fig $1 \mathrm{~b}$.

\section{B. Thermodynamics}

Let us proceed to a discussion of the free energy (4). For the present model, the Boltzmann factors are taken using the energy levels given by 25,30 ,

$$
w_{n}=\sum_{k=0}^{5} g_{n, k} \mathrm{e}^{-\beta \varepsilon_{n, k}} .
$$

Hence, we have reached the following Boltzmann factors

$$
\begin{aligned}
w_{n}= & 2 \mathrm{e}^{\beta\left(\frac{n h}{2}-\frac{J_{z}}{4}\right)}\left\{\left(\mathrm{e}^{\beta J}+2 \mathrm{e}^{-\beta J / 2}\right) \cosh \left(\beta \frac{J_{0}+h_{z}}{2}\right)\right. \\
& \left.+\mathrm{e}^{\beta J_{z}} \cosh \left(\frac{3}{2} \beta\left(J_{0} n+h_{z}\right)\right)\right\},
\end{aligned}
$$

where $n=\{-1,0,1\}$.

Therefore, we can find the system entropy at finite temperature is $\mathcal{S}=-\frac{\partial f}{\partial T}$. Similarly, one can find Heisenberg spin magnetization given by $m_{H}=-\frac{\partial f}{\partial h_{z}}$ and Ising spin magnetization becomes $m_{I}=-\frac{\partial f}{\partial h}$. 
(a)

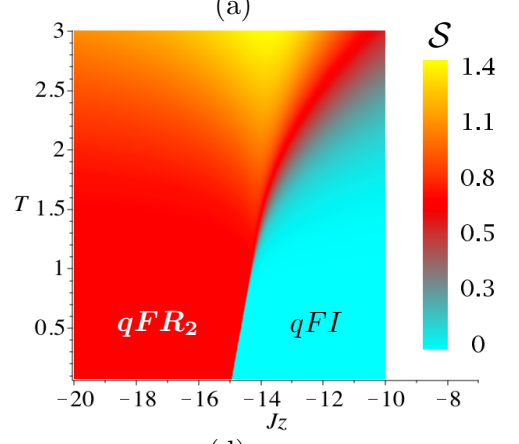

(d)

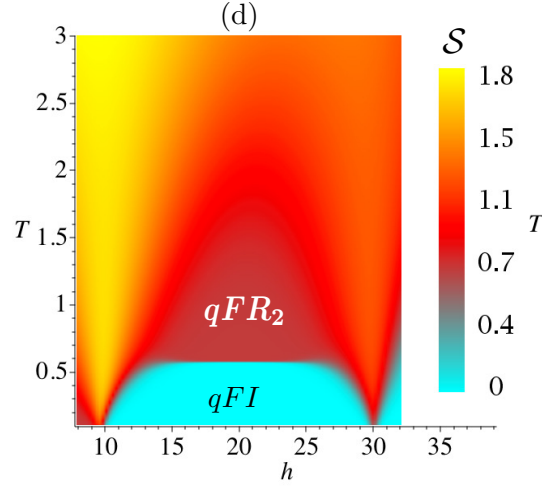

(b)

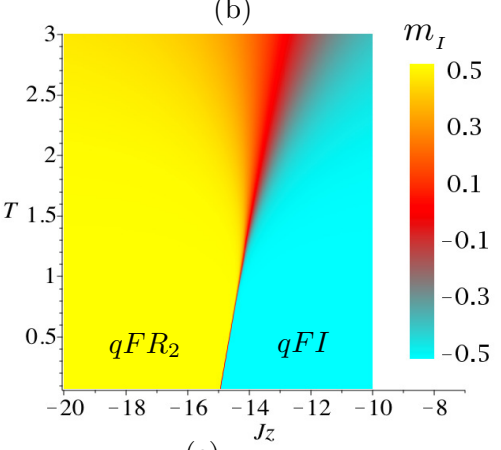

(e)

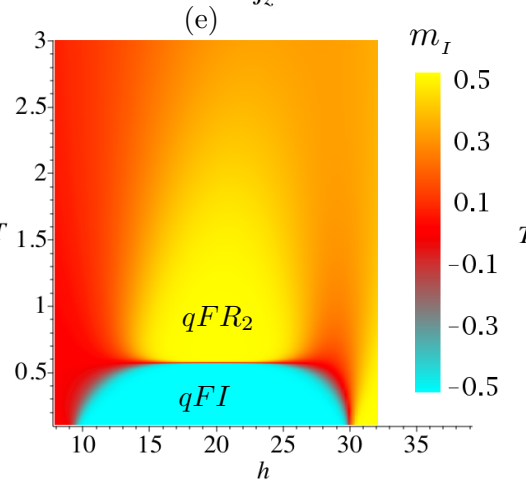

(c)

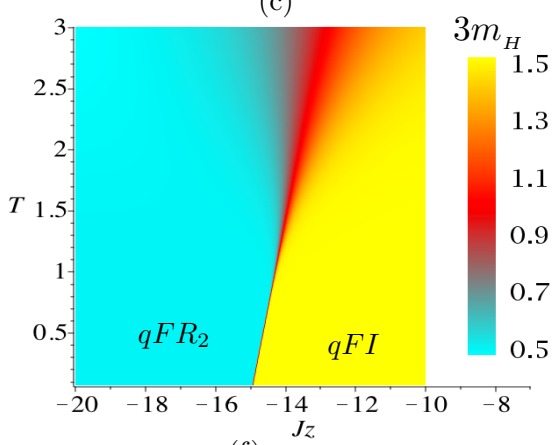

(f)

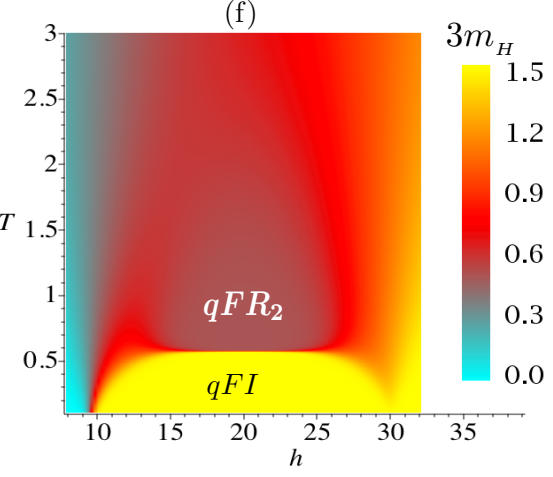

Figure 5: Density plot for Ising-Heisenberg coupled tetrahedral chain in the $J_{z}-T$ plane, assuming fixed $J=-10, J_{0}=-10$ : (a) entropy for $h=20$; (b) Ising spin magnetization for $h=20$; (c) Heisenberg spin magnetization for $h=20$; (d) entropy in the $h-T$ plane for fixed $J_{z}=-14.6$; (e) Ising spin magnetization for $J_{z}=-14.6$; (f) Heisenberg spin magnetization for $J_{z}=-14.6$.

In fig $4 \mathrm{~b}$ we illustrate the density plot of entropy as a function of $J_{z}$ and $h$, for fixed $T=0.6$, and using the same scale of fig 4 a. Here we can observe that entropy follows the vestige of the zero temperature phase diagram. Definitely, the thermal excitation influences the phase boundaries, and all except one, display an increase

Fig 5(left column) reports density plot of entropy in the $J_{z}-T$ plane (panel a) and $h-T$ plane (panel d), for the parameters considered in the caption. Panel (a) for $-15<J_{z} \lesssim-14$ exhibits a pseudo-transition between quasi-phases $q F R_{2}$ and $q F I$, for $J_{z} \gtrsim-14$ the sharp boundary melt. Panel (d) for $15 \lesssim h \lesssim 25$, we observe a sharp boundary between quasi-phases $q F R_{2}$ and $q F I$, for other values of magnetic field the boundaries melt. In fig 5 (middle column) is illustrated the Ising spin magnetization $m_{I}$ in the $J_{z}-T$ plane (panel b), and in the $h-T$ plane (panel e). While the right column reports for Heisenberg spin magnetization $\left(m_{H}\right)$ in the $J_{z}-T$ plane (panel c), and in the $h-T$ plane (panel f). So in all panels the phase boundary is easily identified by sharp boundaries.

In fig $6 \mathrm{a}$ is showed the entropy as a function of $J_{z}$ in the low temperatures region. We can observe the track of frustrated $\left(F R_{2}\right)$ phase which is a macroscopically in entropy around the phase boundaries. The entropy at the boundary between $q F I$ and $q F R_{2}$ (here we add the prefix " $q$ " to name the quasi-phases) holds almost unaltered. Because at zero temperature, residual entropy is continuous, at least from the one-sided limit at the phase boundary.

degenerate state, whose residual entropy is $\mathcal{S}=\ln (2)$. The peak corresponds to the phase boundary between $F R_{2}$ and $S A$, with its respective CRE given by $\mathcal{S}_{c}=$ $\ln (3)$. Here we can see how entropy at finite temperature stretched around discontinuous CRE owing to thermal excitation (see fig 1 $\mathrm{k}$ ). In fig $6 \mathrm{~b}$ entropy is illustrated as a dependence of $J_{z}$, where phases $F R_{2}$ and $F I$ have residual entropy $\mathcal{S}=\ln (2)$ and $\mathcal{S}=0$, respectively. Note that entropy remains almost unaffected at $\mathcal{S}_{c}=\ln (2)$ for $T \lesssim 1$ because the residual entropy at zero-temperature is continuous from the one-sided limit at the critical point (see fig $\sqrt{1 b}$ ). In fig 6, we report entropy as a function of temperature at the interface between $F R_{1}[\mathcal{S}=\ln (2)]$ and $F R_{2}[\mathcal{S}=\ln (2)]$, and $\mathcal{S}_{c}=\ln (3+\sqrt{5})$ gives the corresponding CRE for $J_{z} \lesssim-20$. Whereas for $J_{z}=-15$, the phase boundary joins three phases $F R_{1}, F R_{2}$ and $F I$, at first glance this is similar to fig $6 \mathrm{~b}$. But the CRE at zero temperature is larger than the adjacent phases residual 

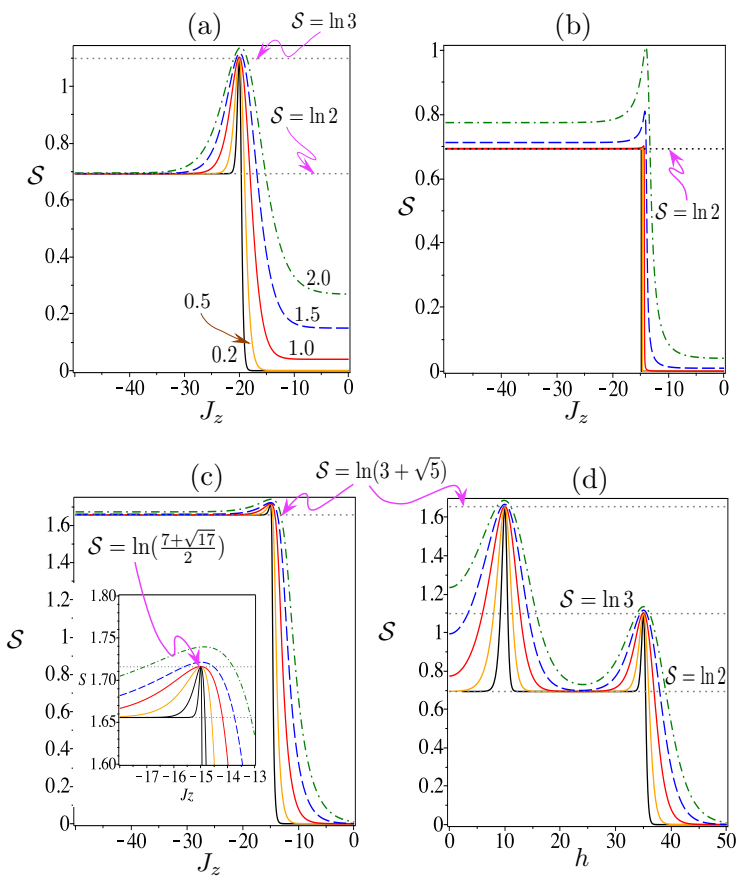

Figure 6: (a-c) Entropy for Ising-Heisenberg coupled tetrahedral chain as a function of $J_{z}$ assuming fixed $J=-10, J_{0}=$ -10 , for a set of temperatures $T=\{0.2,0.5,1.0,1.5,2.0\}$ : (a) for $h=35$; (b) for $h=20$; (c) for $h=10$; (d) entropy as a dependence of $h$ for fixed $J=-10, J_{0}=-10, J_{z}=-20$ and for the same set of temperatures in (a-c).

entropies, which can be obtained using the eq. A6 . Since the degeneracies of each sector are $g_{1,0}^{c}=4, g_{-1,0}^{c}=3$ and $g_{0,0}^{c}=2$, under these circumstances the critical residual entropy becomes $\mathcal{S}_{c}=\ln \left(\frac{7+\sqrt{17}}{2}\right)$. We can observe better this point in a magnified plot in the inner part of the fig 6c. Because of this small peak, the right side curve stretched around CRE for $T>0$, destroying any evidence of pseudo-transition. At last, in fig $6 \mathrm{~d}$ is plotted the entropy as a function of magnetic field $h$. Then we observe a residual entropy between phase boundaries, which are in agreement with the previous plots (panels a and c).

In synthesis, it is worth mentioning that the entropy [fig $6(a$ and $c)$ ] at zero temperature falls for a type of function described in fig 1 , while fig $6 \mathrm{~d}$ falls for a kind of function described in fig.1 (c-d) confirming the absence of pseudo-transition. In contrast, fig $6 \mathrm{p}$ fits into a type of fig $1 \mathrm{~b}$, which means the existence of pseudo-transition.

Fig 7 depicts entropy as a function of temperature assuming fixed parameters $J=-10, J_{0}=$ $-10, \quad h=20$ and for several values $J_{z}=$ $\{-13,-14.1,-14.3,-14.5,-14.6\}$. It is evident to observe a strong change in the entropy curvature at the pseudo-critical temperature. Thus, as the temperature rises, the sharp jump in entropy becomes softer and gradually vanishes. In fig $7 \mathrm{~b}$ is illustrated the correlation length as a temperature dependence, so that it corroborates a sharp and robust peak at pseudo-critical temper-
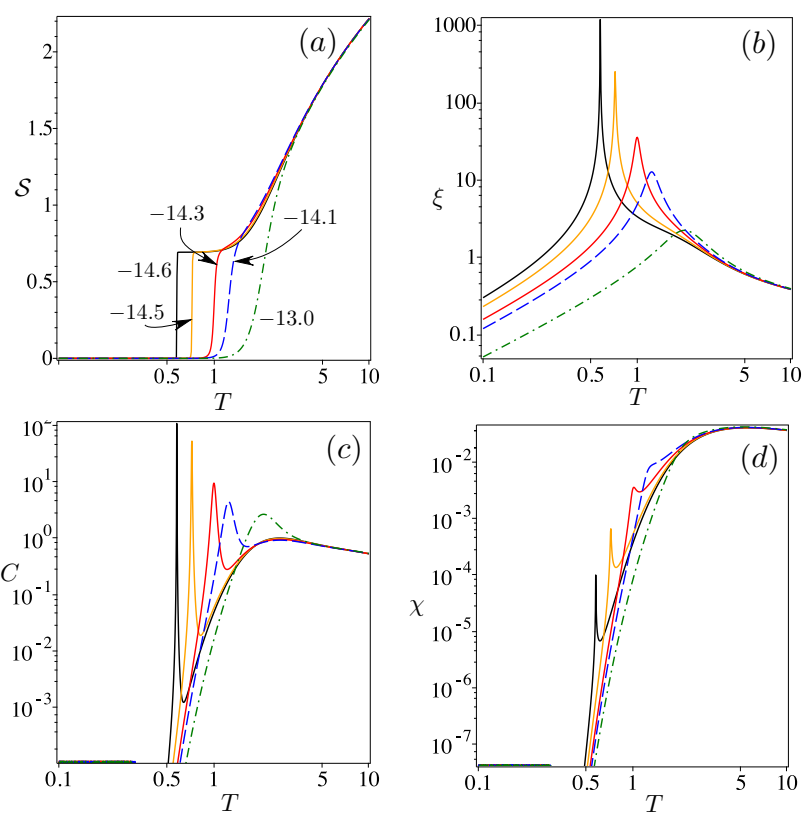

Figure 7: (a) Entropy $\mathcal{S}$ for Ising-Heisenberg coupled tetrahedral chain as a function of temperature, assuming fixed parameters $J=-10, J_{0}=-10, h=20$ and several values of $J_{z}=\{-13,-14.1,-14.3,-14.5,-14.6\}$ (semi-logarithmic plot); (b) correlation length $\xi$ as a dependence of temperature (logarithmic plot); (c) specific heat $C$ against temperature (logarithmic plot); (d) magnetic susceptibility $\chi$ as a function of temperature (logarithmic plot).

ature. In fig $7 \mathrm{k}$ is reported the specific heat as a function of temperature, and once again, we observe a sharp peak at pseudo-critical temperature. Whereas in fig $7 \mathrm{~d}$ is reported the magnetic susceptibility as a function of temperature, which exhibits a small sharp peak. Since the total magnetization at zero temperature is identical for both phases $F R_{2}$ and $F I$ according to $(32)$ and (34), respectively.

\section{ISING-XYZ DIAMOND CHAIN}

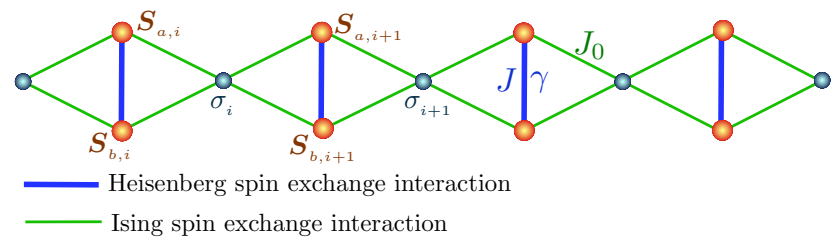

Figure 8: Schematic representation of Ising-XYZ diamond chain. Small spheres $\left(\sigma_{i}\right)$ correspond to Ising spins and large spheres $\left(\boldsymbol{S}_{a(b), i}\right)$ correspond to Heisenberg spins.

Another model we consider here is the Ising-XYZ diamond chain structure, as illustrated in fig, 8 , which was discussed earlier in reference [12 14. Here $\sigma_{i}$ (small spheres) represents the Ising spin- $1 / 2$, and $S_{a(b), i}^{\alpha}$ (large 
spheres) denotes the Heisenberg spin- $1 / 2$, with $\alpha=$ $\{x, y, z\}$. Despite Ising-XXZ diamond chain is frustrated owing to triangular structure and $x y$-isotropy, Ising$\mathrm{XYZ}$ diamond chain also exhibits unfrustrated phase boundary 12 14, because of $x y$-anisotropy. Thus, here we only give a revisiting of Ising-XYZ diamond chain, whose Hamiltonian is expressed by

$$
\begin{aligned}
H=-\sum_{i=1}^{N}[ & J(1+\gamma) S_{a, i}^{x} S_{b, i}^{x}+J(1-\gamma) S_{a, i}^{y} S_{b, i}^{y} \\
& +J_{z} S_{a, i}^{z} S_{b, i}^{z}+J_{0}\left(S_{a, i}^{z}+S_{b, i}^{z}\right)\left(\sigma_{i}+\sigma_{i+1}\right) \\
& \left.+h_{z}\left(S_{a, i}^{z}+S_{b, i}^{z}\right)+\frac{h}{2}\left(\sigma_{i}+\sigma_{i+1}\right)\right]
\end{aligned}
$$

where $J$ corresponds to $x y$-axis exchange interaction and $\gamma$ being the $x y$-anisotropy, $J_{z}$ stands for Heisenberg spins exchange interaction on the $z$-axis. While $J_{0}$ denotes Ising-Heisenberg spin exchange interaction, and $h_{z}(h)$ corresponds to the external magnetic field along the $z$ axis acting on Heisenberg spin (Ising spin), respectively.

\section{A. Zero temperature phase diagram}

Further investigation of the ground state phase diagram has already been found in reference [13, 14]. Below are summarized some of those ground states assuming $n=\sigma_{i}+\sigma_{i+1}$ : is

(i) For sector $n=1(\uparrow \uparrow)$ the first ground state energy

$$
\varepsilon_{1,0}=E_{M F_{2}}=-\frac{J_{z}}{4}-\frac{h}{2}-\sqrt{\left(h_{z}+J_{0}\right)^{2}+\frac{1}{4} J^{2} \gamma^{2}} .
$$

Named as modulated ferromagnetic Heisenberg spin $\left(M F_{2}\right)$ phase. With its corresponding ground state

$$
\left.\left.\left|M F_{2}\right\rangle=\prod_{i=1}^{N}\left(\left.\cos \theta_{1}\right|_{+} ^{+}\right\rangle_{i}+\left.\sin \theta_{1}\right|_{-} ^{-}\right\rangle_{i}\right) \otimes|\uparrow\rangle_{i},
$$

where $\theta_{n}=\frac{1}{2} \tan ^{-1} \frac{J \gamma}{2\left(h_{z}+J_{0} n\right)}$ defined in $-\frac{\pi}{4}<\theta_{n}<\frac{\pi}{4}$.

Another state is the ferrimagnetic (FI) phase, which is given by

$$
\varepsilon_{1,1}=E_{F I}=-\frac{J+h}{2}+\frac{J_{z}}{4},
$$

In fig 9 a we illustrate the density plot entropy as a function of temperature for fixed parameters $J=100$, $\gamma=0.8, J_{z}=24$ and $J_{0}=-24$. The density plot of entropy in low-temperature region shows an indistinguishable boundary between quasi-phases $q M F_{0}$ and $q M F_{2}$. Because, at zero temperature, the ground state energy for both phases $M F_{0}$ and $M F_{2}$ are non-degenerate, which implies that the residual entropy is $\mathcal{S}=0$. Besides, CRE also becomes null according to relation 11 , which is eas- and the corresponding ground state is expressed as

$$
\left.\left.|F I\rangle=\prod_{i=1}^{N} \frac{1}{\sqrt{2}}\left(\left.\right|_{+} ^{-}\right\rangle_{i}+\left.\right|_{-} ^{+}\right\rangle_{i}\right) \otimes|\uparrow\rangle_{i}
$$

(ii) For sector $n=-1(\downarrow \downarrow)$, the ground state energy, becomes

$$
\varepsilon_{-1,0}=E_{M F_{0}}=-\frac{J_{z}}{4}+\frac{h}{2}-\sqrt{\left(h_{z}-J_{0}\right)^{2}+\frac{1}{4} J^{2} \gamma^{2}}
$$

with its respective modulated ferromagnetic $\left(M F_{0}\right)$ state, given by

$$
\left.\left.\left|M F_{0}\right\rangle=\prod_{i=1}^{N}\left(\left.\cos \theta_{-1}\right|_{+} ^{+}\right\rangle_{i}+\left.\sin \theta_{-1}\right|_{-} ^{-}\right\rangle_{i}\right) \otimes|\downarrow\rangle_{i} .
$$

Now assuming $h_{z}=h$, the ground state energy at the interface between $M F_{0}$ and $M F_{2}$ must coincide (see fig 9 , implying that there is a critical magnetic field given by

$$
h_{c}=\frac{\left(\gamma^{2}-1\right)+2 J_{z} J+4 J_{0}^{2}-J_{z}^{2}}{4 J+8 J_{0}-4 J_{z}} .
$$

For $h<h_{c}$ the system is in $M F_{0}$ state, and for $h>h_{c}$ the system becomes in $M F_{2}$ state.

\section{B. Thermodynamics}

Next, let us take a look at the thermodynamics of the model. Thus, the free energy (4) for the Ising-XYZ diamond chain was also obtained in reference [13, 14], where the Boltzmann factors were given by

$$
w_{n}=2 \mathrm{e}^{\frac{\beta n h}{2}}\left[\mathrm{e}^{-\frac{\beta J_{z}}{4}} \operatorname{ch}\left(\frac{\beta J}{2}\right)+\mathrm{e}^{\frac{\beta J_{z}}{4}} \operatorname{ch}\left(\beta \Delta_{n}\right)\right],
$$

with $\Delta_{n}=\sqrt{\left(h_{z}+J_{0} n\right)^{2}+\frac{1}{4} J^{2} \gamma^{2}}$.

ily verified on the density plot of entropy.

However, the density plot of Heisenberg spin magnetization $\left(m_{H}\right)$ in the plane $h-T$ is reported in fig $9 \mathrm{~b}$. Doubtless, the boundary between quasi-phases $q M F_{2}$ and $q F M_{0}$ shows a distinguishable region. At $T=0$ and $h<h_{c}=13.063045$, the Heisenberg spins are parallel ordered with greater probability pointing up. The maximum magnetization per spin is $m_{H} \sim 0.3$, which is pictorially denoted as effective canting spin (yellow re- 
(a)

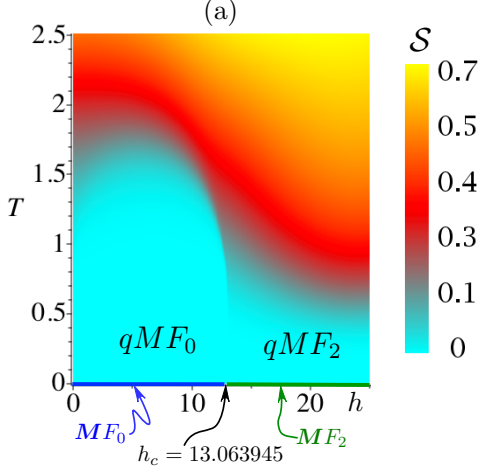

(b)

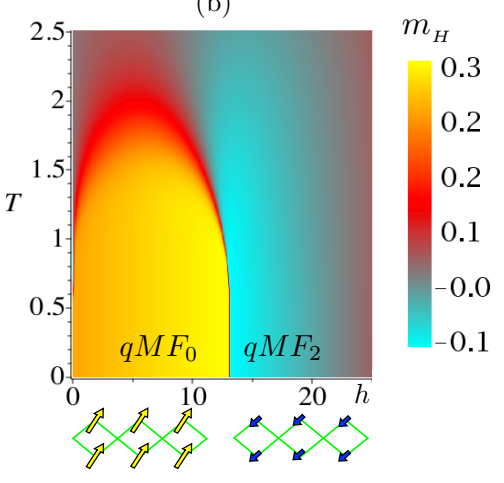

(c)

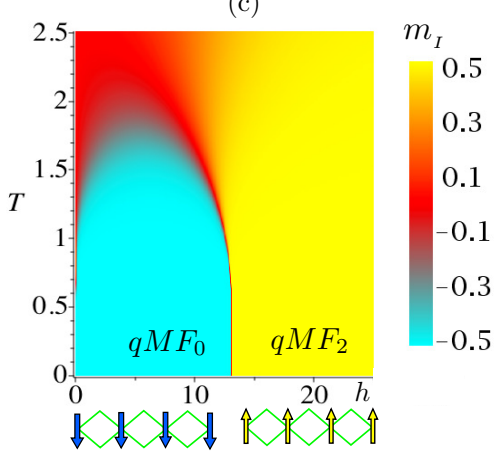

Figure 9: Density plot for Ising-XYZ diamond chain assuming fixed parameters $J=100, \gamma=0.8, J_{z}=24$ and $J_{0}=-24$ in the $h-T$ plane: (a) entropy; (b) Heisenberg spin magnetization; (c) Ising spin Magnetization.

gion). Similarly, for $h>h_{c}=13.063945$ the Heisenberg spin magnetization becomes negative $m_{H} \sim-0.1$, pictorially illustrated by effective canting spin down (cyan region). For more detailed information concerning Heisenberg spin magnetization, we refer the readers to ref. [10. In a similar way the fig 9 e displays the density plot of Ising spin magnetization, for $h<h_{c}$ the magnetization is nearly $m_{I}=-0.5$, which means most of the Ising spins are aligned downward. While for $h>h_{c}$ most Ising spins are pointing up, aligning with the external magnetic field. Consequently, this behavior could be easily misinterpreted as a true phase transition. Although, we do not expect a genuine phase transition at finite temperature, because all free energy derivatives are analytical. It is noteworthy that, at finite temperature, there is no critical magnetic field. However, only a pseudo-critical magnetic field $h_{p} \lesssim h_{c}$, which vanishes roughly around $T \sim 1.0$, for temperature $T \gtrsim 1.0$, the system becomes a standard disordered system predominantly.

Fig $10 \mathrm{a}$ illustrates the entropy as a function of $h$, for a set of temperature values $T=\{0.3,0.5,1.0,1.5,2.0\}$. It can be seen from the curve, that there is a sudden change for $T \lesssim 1.0$, this one corresponds to pseudotransition at $h=13.063945$. Therefore, for any region or quasi-phase, the entropy vanishes when $T \rightarrow 0$. Similarly, in fig $10 \mathrm{~b}$ entropy is plotted as a dependence of $h$, where we observe a continuous sudden change to the magnetic field $h=12.8$. Again, as soon as temperature decreases, the entropy vanishes according to fig 1 . However, for $h \approx 39$ corresponds to the phase boundary between $F I$ and $M F_{2} 12$ 14 with a critical residual entropy $\mathcal{S}_{c}=\ln (2)$, and obviously in this boundary there is no pseudo-transition (see fig $1 \mathrm{~d}$ ). Furthermore, in fig 10 d, entropy against $\gamma$ is plotted for $h=18$ and $h=12$. For temperature $T \lesssim 1$, a continuous sudden change appears, showing the pseudo-transition. Entropy vanishes when $T \rightarrow 0$, because the CRE becomes null, which is in accordance with fig 1 a. In reference [10, 12, 14], the reader can find other detailed discussions concerning this model.
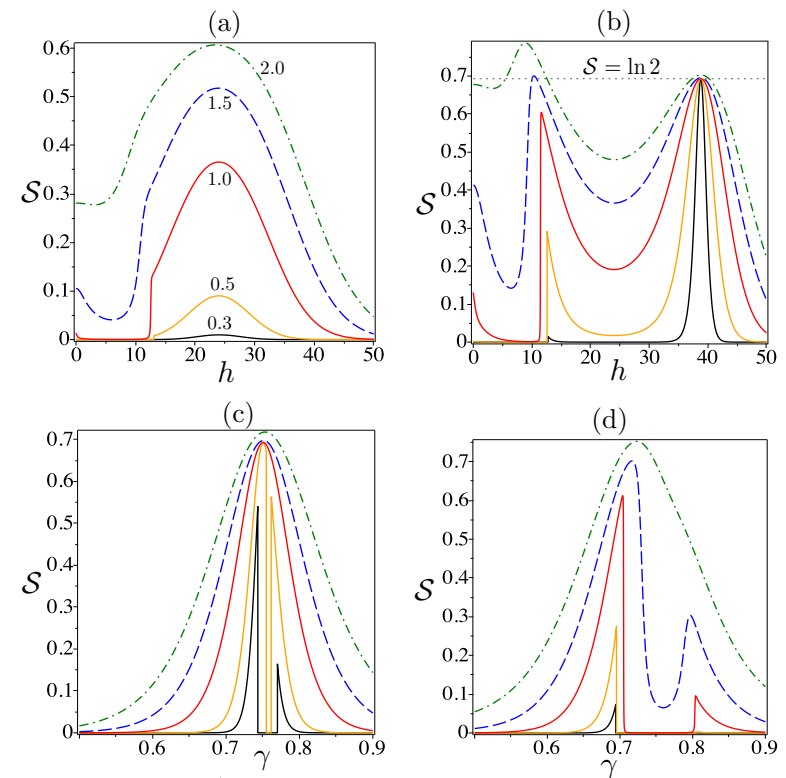

Figure 10: Entropy for Ising-XYZ diamond chain considering fixed parameters $J=100, J_{z}=24, J_{0}=-24$ and several values of temperatures $T=\{0.3,0.5,1.0,1.5,2.0\}$ : (a) as a function of $h$ considering $\gamma=0.8$; (b) as a function of $h$ assuming $\gamma=0.7$; (c) as a function of $\gamma$ considering $h=18$; (d) as a function of $\gamma$ for a fixed $h=12$.

\section{CONCLUSIONS.}

Although few one-dimensional models exhibit the phase transition [4 7], this phenomenon is related to some null elements of the transfer-matrix, which leads to a non-analytic free energy. Nevertheless, pseudo-critical temperatures have recently been investigated in onedimensional spin models [10, 12]. Therefore, there are several models exhibiting pseudo-transitions, such as IsingHeisenberg spin models with a variety of structures 13 , 14, 16-18].

We propose here a relationship between critical residual entropy at zero temperature and pseudo-transition. 
In general, residual entropy increases at the interface where the phase transition occurs at a critical point. Which means the system increases its ground state degeneracy in the interface compared to adjacent states. In contrast, there are few cases where CRE remains equal to the largest residual entropy of neighboring states, here $\mathrm{CRE}$ is given by $\mathcal{S}_{c}=\ln \left[\max \left(g_{1,0}, g_{-1,0}\right)\right]$. So our main result dwells in a simple argument to recognize a pseudotransition. If the zero temperature residual entropy is continuous at least from the one-sided limit at critical point, then analytical free energy keep in sight the evidences of a pseudo-transition at finite temperature around critical point. In order to show the aforementioned property, we considered two Ising-Heisenberg spin models: one frustrated model in a coupled tetrahedral chain and another unfrustrated diamond chain.

Finding pseudo-transition in more realistic systems, like the quantum Heisenberg model, would be a fascinating investigation. Nevertheless, this would be a cumbersome numerical task at finite temperature. However, searching for residual entropy at zero temperature should be an easier task than studying the complete thermodynamics of the model. Only after this analysis would it be possible to study thermodynamics for a particular condition previously analyzed at zero temperature. In this sense, it would be interesting whether the condition of the phase boundary entropy still holds. Assuming our argument is still valid, we can apply this condition at zero temperature and look for continuity of residual entropy, which would be a more manageable task than studying the complete thermodynamics of the model.

\section{Acknowledgments}

This work was partially supported by ICTP and Brazilian agencies CNPq and FAPEMIG.

\section{Appendix A: Discontinuous residual entropy}

When CRE is larger than its neighboring residual entropy at zero temperature, it means a discontinuous residual entropy because of $G_{c}>\max \left(g_{1,0}, g_{-1,0}\right)$. Then, to obtain the residual entropy we can use the free energy (4) in the limit $T \rightarrow 0$. Below we get for some particular cases, the residual entropy at zero temperature.

1. Phase boundary between states of sector $n=0$ and $n= \pm 1$

Here let us consider the sector $n=1$ and $n=0$, where the lowest energies are given by $\varepsilon_{1,0}(x)$ and $\varepsilon_{0,0}(x)$. Then the phase boundary occurs when $\varepsilon_{1,0}\left(x_{c}\right)=\varepsilon_{0,0}\left(x_{c}\right)=\varepsilon_{c}$ with corresponding degeneracies $g_{1,0}^{c}$ and $g_{-1,0}^{c}$. Eventually, we could have $g_{1,0}^{c} \geqslant g_{1,0}$ and $g_{0,0}^{c} \geqslant g_{0,0}$. The lowest energy $\varepsilon_{-1,0}(x)$ in sector $n=-1$, will be strictly higher than $\varepsilon_{c}\left(\varepsilon_{-1,0}>\varepsilon_{c}\right)$, what means $w_{-1} / w_{0} \rightarrow 0$ and $w_{-1} / w_{1} \rightarrow 0$ when $T \rightarrow 0$. So the free energy in (4) at sufficiently low temperature becomes

$$
f=-\frac{1}{\beta} \ln \left[\frac{1}{2}\left(g_{1,0}^{c}+\sqrt{\left(g_{1,0}^{c}\right)^{2}+4\left(g_{0,0}^{c}\right)^{2}}\right) \mathrm{e}^{-\beta \varepsilon_{c}}\right] .
$$

Consequently, the CRE turns in

$$
\mathcal{S}_{c}=\ln \left[\frac{1}{2}\left(g_{1,0}^{c}+\sqrt{\left(g_{1,0}^{c}\right)^{2}+4\left(g_{0,0}^{c}\right)^{2}}\right)\right],
$$

where the critical degeneracy results in $G_{c}=\frac{1}{2}\left(g_{1,0}^{c}+\right.$ $\left.\sqrt{\left(g_{1,0}^{c}\right)^{2}+4\left(g_{0,0}^{c}\right)^{2}}\right)$.

We can observe the critical degeneracy is strictly larger than its adjacent degeneracies: $G_{c}>g_{1,0}$ and $G_{c}>g_{0,0}$. The residual entropy is reported schematically in fig 1 . Note that the, residual entropy $\mathcal{S}_{a}$ and $\mathcal{S}_{b}$ denote in general a residual entropy between adjacent states.

For sector $n=-1$ and $n=0$, the result of free energy will be equivalent to the previous case. Therefore we can obtain merely by exchanging $\varepsilon_{1,0}\left(x_{c}\right) \rightarrow \varepsilon_{-1,0}\left(x_{c}\right)$, in expression A2.

\section{Phase boundary lying in a single sector}

Assuming that occurs a phase transition between two states with energies $\varepsilon_{1,0}(x)$ and $\varepsilon_{1,1}(x)$, then the critical energy is given by $\varepsilon_{1,0}\left(x_{c}\right)=\varepsilon_{1,1}\left(x_{c}\right)=\varepsilon_{c}$ at $T=0$. In general, it is possible that some additional states can coincide at the phase boundary, then the degeneracies can be eventually expressed satisfying the condition $g_{1,0}^{c} \geqslant g_{1,0}$ and $g_{1,1}^{c} \geqslant g_{1,1}$. Therefore, all other energy levels must be higher than $\varepsilon_{c}$, so when $T \rightarrow 0$, the spectral energy in other sectors can be neglected $\left(w_{0} / w_{1} \rightarrow 0\right.$ and $\left.w_{-1} / w_{1} \rightarrow 0\right)$. Hence, the free energy in the low temperature limit is expressed as

$$
\begin{aligned}
f & =-\frac{1}{\beta} \ln \left(w_{1}\right)=-\frac{1}{\beta} \ln \left(g_{1,0}^{c} \mathrm{e}^{-\beta \varepsilon_{c}}+g_{1,1}^{c} \mathrm{e}^{-\beta \varepsilon_{c}}\right) \\
& =-\frac{1}{\beta} \ln \left[\left(g_{1,0}^{c}+g_{1,1}^{c}\right) \mathrm{e}^{-\beta \varepsilon_{c}}\right] .
\end{aligned}
$$

Whereas, the corresponding critical residual entropy, reduces to

$$
\mathcal{S}_{c}=\ln \left(g_{1,0}^{c}+g_{1,1}^{c}\right)
$$

Thereby, the critical degeneracy is given by $G_{c}=\left(g_{1,0}^{c}+\right.$ $\left.g_{1,1}^{c}\right)$.

Once again, the CRE is strictly higher than any residual entropy of adjacent states, because $G_{c}>g_{1,0}^{c}$ and $G_{c}>g_{1,1}^{c}$. A schematic representation of this type of CRE is illustrated in fig 1 .

\section{Phase boundary lying in three sectors}

When three sectors can constitute the phase boundary, the states with energies $\varepsilon_{1,0}(x), \varepsilon_{0,0}(x)$ and $\varepsilon_{-1,0}(x)$, can 
coexist for a particular $x_{c}$ (a critical point). So we must assume $\varepsilon_{1,0}\left(x_{c}\right)=\varepsilon_{0,0}\left(x_{c}\right)=\varepsilon_{-1,0}\left(x_{c}\right)=\varepsilon_{c}$, and the respective degeneracies are $g_{1,0}^{c}, g_{0,0}^{c}$ and $g_{-1,0}^{c}$. In this case, the free energy becomes

$$
\begin{aligned}
f= & -\frac{1}{\beta} \ln \left[\frac { 1 } { 2 } \left(g_{1,0}^{c}+g_{-1,0}^{c}+\right.\right. \\
& \left.\left.+\sqrt{\left(g_{1,0}^{c}-g_{-1,0}^{c}\right)^{2}+4\left(g_{0,0}^{c}\right)^{2}}\right) \mathrm{e}^{-\beta \varepsilon_{c}}\right] .
\end{aligned}
$$

Finally, the CRE reads

$$
\mathcal{S}_{c}=\ln \left[\frac{1}{2}\left(g_{1,0}^{c}+g_{-1,0}^{c}+\sqrt{\left(g_{1,0}^{c}-g_{-1,0}^{c}\right)^{2}+4\left(g_{0,0}^{c}\right)^{2}}\right)\right] .
$$

Then the critical degeneracy is $G_{c}=\frac{1}{2}\left(g_{1,0}^{c}+g_{-1,0}^{c}+\right.$ $\left.\sqrt{\left(g_{1,0}^{c}-g_{-1,0}^{c}\right)^{2}+4\left(g_{0,0}^{c}\right)^{2}}\right)$.
Similar to the previous cases, the CRE is strictly larger than its adjacent states residual entropies, because $G_{c}>$ $g_{1,0}^{c}, G_{c}>g_{0,0}^{c}$ and $G_{c}>g_{-1,0}^{c}$.

In all aforementioned cases, the CRE inevitably exhibits a jump-point discontinuity or a point discontinuity at $x_{c}$ (see fig, 1k-d). Because the CRE is strictly larger than its adjacent states residual entropy.
[1] L. van Hove, Physica 16,137 (1950)

[2] J. A. Cuesta and A. Sanchez, J. Stat. Phys. 115, 869 (2004)

[3] N. D. Mermin and H. Wagner, Phys. Rev. Lett. 17, 1133 (1966)

[4] C. Kittel, Am. J. Phys. 37, 917(1969)

[5] S. T. Chui and John D. Weeks, Phys. Rev. B 23, 2438 (1981)

[6] T. Dauxois and M. Peyrard, Phys. Rev. E 51, 4027 (1995)

[7] P. Sarkanych, Y. Holovatch and R. Kenna, Phys. Lett. A 381, 3589 (2017)

[8] F. Ninio, J. Phys. A: Math Gen 91281 (1976)

[9] P. N. Timonin, J. Exp. Theor. Phys. 113, 251 (2011)

[10] I. M. Carvalho, J. Torrico, S. M. de Souza, O. Rojas, O. Derzhko, Ann. Phys. 402, 45 (2019)

[11] I. M. Carvalho, J. Torrico, S. M. de Souza, M. Rojas, O. Rojas, J. Magn. Magn. Mats. 465, 323 (2018)

[12] S. M. de Souza and O. Rojas, Sol. State. Comm. 269, 131 (2018)

[13] J. Torrico, M. Rojas, S. M. de Souza, O. Rojas and N. S. Ananikian, Eur. Phys. Lett. 108, 50007 (2014)

[14] J. Torrico, M. Rojas, S. M. de Souza and O. Rojas, Phys. Lett. A 380, 3655 (2016)

[15] J Strečka, Acta Phys. Pol. A 137, 610 (2020); arXiv:2002.06942
[16] L. Galisova and J. Strecka, Phys. Rev. E 91, 022134 (2015)

[17] O. Rojas, J. Strečka and S.M. de Souza, Sol. State. Comm. 246, 68 (2016)

[18] J. Strecka, R. C. Alecio, M. Lyra and O. Rojas, J. Magn. Magn. Mats. 409, 124 (2016)

[19] ] I. Syozi, Prog. Theor. Phys. 6, 341 (1951); M. Fisher, Phys. Rev. 113, 969 (1959); O. Rojas, J. S. Valverde, S. M. de Souza, Physica A 388, 1419 (2009); J. Strečka, Phys. Lett. A 374, 3718 (2010); O. Rojas, S. M. de Souza, J. Phys. A: Math. Theor. 44, 245001 (2011)

[20] O. Rojas, Acta Phys. Pol. A 137, 933 (2020).

[21] J. Stewart, Single Variable Calculus early transcendentals, 7th Ed. Brooks/Cole (2012)

[22] K. Karlova, J. Strecka, T. Madaras, Physica B 488, 49 (2016)

[23] M. Mambrini, J. Trebosc and F. Mila, Phys. Rev. B 59 13806 (1999)

[24] O. Rojas, F. C. Alcaraz, Phys. Rev. B 67, 174401 (2003)

[25] L. Galisova, Phys. Rev. E 96, 052110 (2017)

[26] V. Ohanyan, Cond. Matt. Phys., 12, 343 (2009)

[27] D. Antonosyan, S. Bellucci, V. Ohanyan, Phys. Rev. B 79, 014432 (2009)

[28] O. Rojas, J Strečka, O. Derzhko, S. M. de Souza, J. Phys.: Condens. Matter 32, 035804 (2020) 